The Astrophysical Journal, 482:870-873, 1997 June 20

(C) 1997. The American Astronomical Society. All rights reserved. Printed in U.S.A.

\title{
OBSERVATIONS OF TWO MILLISECOND PULSARS IN THE GLOBULAR CLUSTER NGC 5904
}

\author{
S. B. ANDERSON ${ }^{1}$ AND A. WOLSZCZAN \\ Department of Astronomy and Astrophysics, Pennsylvania State University, University Park, PA 16802 \\ AND \\ S. R. Kulkarni and T. A. Prince \\ Division of Physics, Mathematics, and Astronomy, California Institute of Technology, Pasadena, CA 91125 \\ Received 1996 April 8; accepted 1997 January 17
}

\begin{abstract}
We report on $5 \mathrm{yr}$ of pulsar observations of the globular cluster NGC 5904 (M5). A timing model has been obtained for the two known pulsars, and a deep search for additional faint isolated pulsars has lowered the detection flux limit to $40 \mu \mathrm{Jy}$ without further detection. We discuss the details and implications of the phase coherent timing models for PSR B1516+02A and PSR B1516+02B. Of particular interest for the binary PSR B1516 +02B are the observed negative period derivative due to acceleration in the gravitational field of M5 and a detection of orbital precession.

Subject headings: binaries: close - globular clusters: individual (M5) pulsars: individual (PSR B1516+02A, PSR B1516+02B)
\end{abstract}

\section{INTRODUCTION}

Observations of globular clusters over the last decade have revealed a surprisingly large population of radio pulsars. The unique environment found in these old stellar systems produces a class of pulsars that is significantly different from the more populous collection of field pulsars. While the majority of field pulsars are characterized by long spin periods $\left(P_{\text {median }} \simeq 0.62 \mathrm{~s}\right)$ and high magnetic fields $\left(B \simeq 10^{12} \mathrm{G}\right)$, the globular cluster population is characterized by spin periods and magnetic fields several orders of magnitude lower, i.e., $P_{\text {median }} \simeq 5.4 \mathrm{~ms}$ and $B \simeq 10^{8-10} \mathrm{G}$, similar to field millisecond pulsars (Phinney \& Kulkarni 1994). The study of this distinct population provides important information on pulsar formation and evolution as well as the structure and dynamics of globular clusters. In addition, the study of individual exotic binaries formed in the dense central cores of clusters provides precise neutron star mass measurements as well as information on the various stellar interactions operative in globular clusters. For example, PSR B1516+02B appears to be spinning up, an effect seen for five other globular cluster pulsars: PSR $\mathrm{B} 2127+11 \mathrm{~A}$ and D in M15 (Wolszczan et al. 1989b; Anderson 1992), PSR B0021-72C and D in 47 Tucanae (Robinson et al. 1995), and PSR B1744-24A in Terzan 5 (Nice \& Thorsett 1992). This phenomenon has been attributed to acceleration of the pulsars in the gravitational field of their respective clusters (Phinney 1993).

Here we present the results obtained from observations of M5 over the $5 \mathrm{yr}$ period following the discovery of PSR B1516+02A and B (Wolszczan et al. 1989a). In $\S 2$ we describe the observations made with the $305 \mathrm{~m}$ Arecibo telescope. In $\S 3$ we delineate a search for additional faint pulsars. In $\S 4$ we discuss the timing results, and in $\S 5$ we summarize our findings.

\section{OBSERVATIONS}

The $305 \mathrm{~m}$ Arecibo radio telescope was used to make 103 observations of M5 during the $5 \mathrm{yr}$ period from 1989 April

\footnotetext{
${ }^{1}$ Currently at Division of Physics, Mathematics, and Astronomy, California Institute of Technology, Pasadena, CA 91125; sba@srl.caltech.edu.
}

19 to 1994 July 25 , with an average duration of 72 minutes. The observations were taken at a central frequency of 430 $\mathrm{MHz}$ with a $10 \mathrm{MHz}$ receiver bandwidth and directed toward the optical center of the cluster. Both senses of circular polarization were sampled at an effective rate of 1.974 $\mathrm{kHz}$ using the Arecibo correlation spectrometer with 128 lags and three-level quantization (NAIC 1989). The resulting autocorrelation functions were recorded on magnetic tape along with precise time reference information. The start time for each observation was referenced to Coordinated Universal Time (UTC/NIST) via the Global Positioning System of satellites with a $1 \mu \mathrm{s}$ accuracy. This recording scheme allowed for the simultaneous timing of both PSR B1516+02A and B as well as searches for additional faint pulsars (see Anderson 1992 for additional details of the search and timing procedures).

\section{FAINT PULSAR SEARCH}

In order to search for pulsars fainter than PSR $\mathrm{B} 1516+02 \mathrm{~A}$ and $\mathrm{B}(\sim 300$ and $\sim 500 \mu \mathrm{Jy}$, respectively $)$ multiple observations were incoherently combined. A sequence of 73 observations, selected on the basis of minimal radio frequency interference and constituting some $88 \mathrm{hr}$ of telescope time, were individually Doppler corrected to the solar system barycenter and summed. This "stack" search reduced the flux detection limit to $40 \mu \mathrm{Jy}$ (4.7 times lower than that of the single transit search that discovered PSR B1516+02A and B). Because of the changing apparent pulse period of a binary pulse, such a "stack" search is only sensitive to isolated pulsars. However, if the luminosity function for single radio pulsars in M5 is similar to the $L^{-1}$ (per unit logarithm) observed for globular cluster pulsars in M15 (Anderson 1992) and field pulsars (Dewey et al. 1985; Lyne, Manchester, \& Taylor 1985) then about five additional isolated pulsars should have been detected, whereas none were found.

An estimate of the statistical significance of this nondetection may be obtained from the Kolmogorov-Smirnov test. In particular, the confidence with which one may assert the null hypothesis, viz., that the luminosity function for pulsars in M5 is proportional to $L^{-1}$, is only $3.7 \%$. While of 
limited statistical significance, this may indicate that the globular cluster pulsar luminosity function depends on the particular parameters of individual clusters, perhaps via a dependence of the distribution of magnetic fields on the various accretion mechanisms responsible for recycling pulsars in globular clusters. This survey represents the first deep search of an average central density cluster. However, observations of several other "ordinary" clusters, e.g., M13 and M53 (Kulkarni et al. 1991), have been made by us that will help constrain any such variation in the luminosity function.

\section{TIMING RESULTS}

Timing models for PSR B1516+02A and B were obtained in an iterative procedure that involved folding the "raw" data to generate pulse profiles, obtaining topocentric time of arrival (TOA) measurements via cross correlation of these profiles with a template, performing a least-squares fit of the TOAs (Fig. 1) to a timing model using the TEMPO software package (Taylor \& Weisberg 1989), and updating the template profiles.

The association of PSR B1516+02A and B with the globular cluster M5 is based on the spatial coincidence $\left(<40^{\prime \prime}\right.$ from the optical center, comparable to the core radius of $24^{\prime \prime}$ ) and an observed column density of free electrons that is consistent with the value predicted by models of the interstellar electron distribution (Taylor \& Cordes 1993). In addition, the negative period derivative observed for PSR B1516+02B is most naturally attributed to acceleration in the cluster gravitational field (see $\S 4.2$ ). The pulse profiles for PSR B1516+02A and B are shown in Figure 2, and the timing models are presented in Table 1.

\subsection{PSR $B 1516+02 A$}

PSR B1516 $+02 \mathrm{~A}$ is an isolated $5.55 \mathrm{~ms}$ pulsar located at a projected distance from the cluster center of 1.2 core radii. The timing data for PSR B1516+02A consist of 298 integrations of 23 minutes each, with a resulting TOA precision of $90 \mu$ s. The observed ratio $\dot{P} / P=7.4 \times 10^{-18} \mathrm{~s}^{-1}$ is larger than the maximum cluster acceleration, $\max \left|a_{l}\right| / c=4$

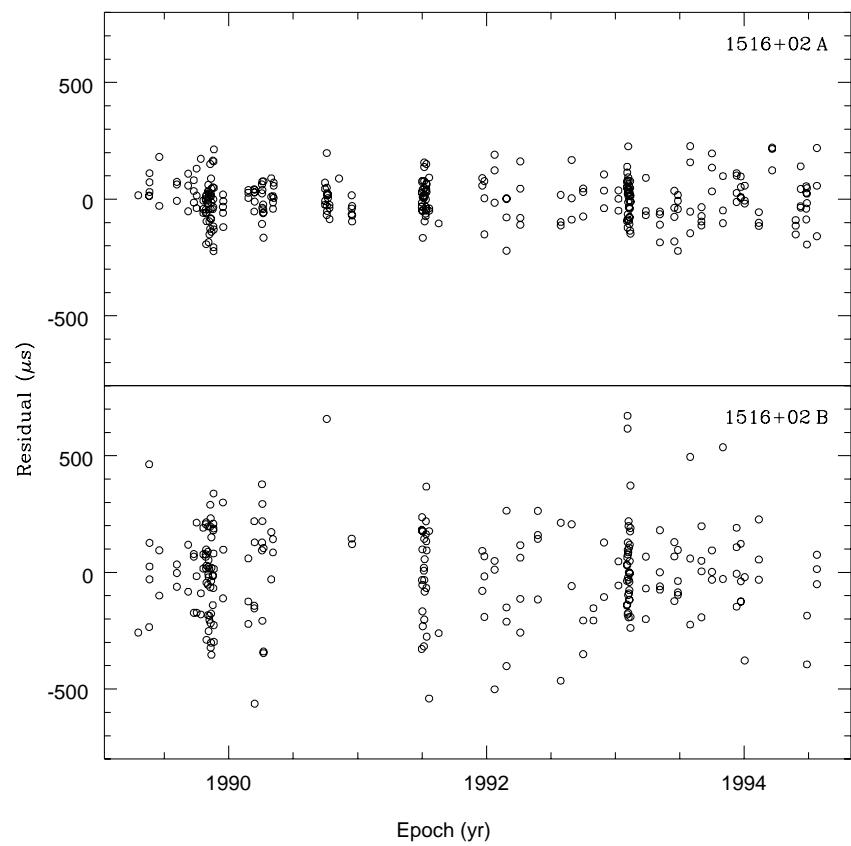

FIG. 1.-Postfit timing residuals for PSR B1516+02A and B. The data correspond to 23 minute integrations and have an uncertainty of $90 \mu \mathrm{s}$ for PSR B1516 + 02A and $200 \mu$ s for PSR B1516+02B.

$\times 10^{-18} \mathrm{~s}^{-1}$, at PSR B1516+02A's projected radius (Phinney 1993). Consequently, the limits on the intrinsic $\dot{P}$ imply a characteristic age between 1.4 and $4.7 \times 10^{9} \mathrm{yr}$, and a magnetic field strength between 3 and $6 \times 10^{8} \mathrm{G}$. Interestingly, the column density toward PSR B1516+02A is larger than that toward PSR B1516+02B (Table 1) even though, as argued below, PSR B1516+02B is behind the cluster and accelerating toward us. This may indicate a gradient in the column density toward M5 of $\sim 0.02 \mathrm{~cm}^{-3}$ pc arcsec ${ }^{-1}$, similar to the $0.1 \mathrm{~cm}^{-3} \mathrm{pc} \operatorname{arcsec}^{-1}$ observed toward M15 (Anderson 1992), and consistent with a spectrum of inhomogeneities in the intervening interstellar

TABLE 1

TIMING PARAMETERS FOR PSR B1516+02A AND B

\begin{tabular}{|c|c|c|}
\hline Parameter & PSR B1516+02A & PSR B1516+02B \\
\hline Period (ms) & $5.553592524205(2)$ & $7.946940657879(21)$ \\
\hline Period derivative $\left(\mathrm{s} \mathrm{s}^{-1}\right)$. & $4.122(6) \times 10^{-20}$ & $-0.333(26) \times 10^{-20}$ \\
\hline Right ascension $(\mathrm{J} 2000)$.. & $15^{\mathrm{h}} 18^{\mathrm{m}} 33^{\mathrm{s}} .3176(6)$ & $15^{\mathrm{h}} 18^{\mathrm{m}} 31^{\mathrm{s}} .458(3)$ \\
\hline Cluster offset ${ }^{\mathrm{b}} \ldots \ldots \ldots$ & -6.5 & $-34 " .4$ \\
\hline Declination (J2000) & $02^{\circ} 05^{\prime} 27^{\prime \prime} .55(3)$ & $02^{\circ} 05^{\prime} 15^{\prime \prime} 47(5)$ \\
\hline Cluster offset $^{\mathrm{b}} \ldots \ldots$ & $+29 " 9$ & $+17 " .8$ \\
\hline Radial offset ${ }^{\mathrm{b}}$. & 30.5 & 38.7 \\
\hline Epoch (MJD) . & 48,445 & 48,445 \\
\hline Dispersion measure $\left(\mathrm{cm}^{-3} \mathrm{pc}\right) \ldots \ldots$ & $30.08(5)$ & $29.47(11)$ \\
\hline 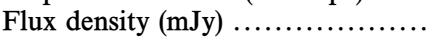 & $\sim 0.5$ & $\sim 0.3$ \\
\hline Orbital period (days) .............. & $\ldots$ & $6.858454(3)$ \\
\hline Projected semimajor axis (ls) ....... & $\ldots$ & $3.04859(7)$ \\
\hline Eccentricity ............ & $\ldots$ & $0.13784(3)$ \\
\hline Longitude of periastron. & $\ldots$ & $-0.321(15)$ \\
\hline Orbital precession $\left(\mathrm{yr}^{-1}\right)^{\mathrm{a}} .$. & $\ldots$ & 0.015 \\
\hline Epoch of periastron (MJD) ......... & $\ldots$ & 48441.8144(2) \\
\hline Mass function $\left(M_{\odot}\right) \ldots \ldots \ldots \ldots \ldots \ldots$ & $\ldots$ & $0.00064674(3)$ \\
\hline
\end{tabular}

\footnotetext{
NotE.-Numbers in parentheses are twice the formal uncertainties in the last digit quoted.

${ }^{\text {a }}$ See text for uncertainty.

${ }^{b}$ Positional offsets from the optical center of M5: $15^{\mathrm{h}} 18^{\mathrm{m}} 33.75,02^{\circ} 04^{\prime} 57^{\prime \prime} 7$ (J2000) with an 0".26 error circle; Shawl \& White 1986.
} 


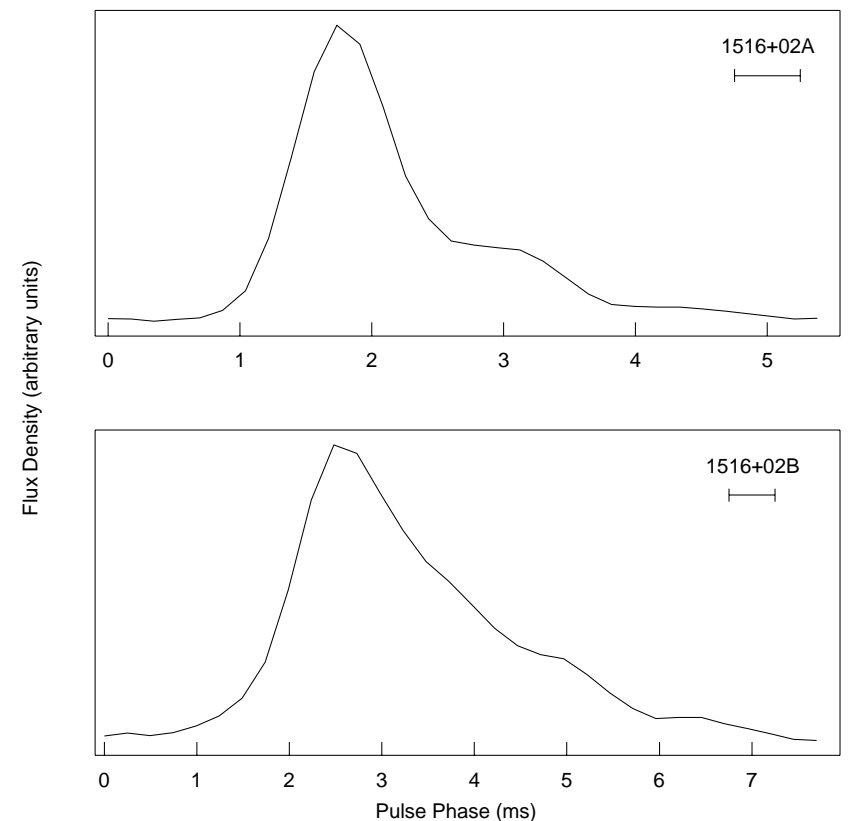

FIG. 2.-Pulse profiles for PSR B1516+02A and B. The $500 \mu$ s temporal resolution is denoted by the horizontal error bars.

medium extending out to length scales of $\gtrsim 10^{17} \mathrm{~cm}$ (Rickett 1990).

\subsection{PSR $B 1516+02 B$}

PSR B1516+02B is a binary $7.95 \mathrm{~ms}$ pulsar with a position offset from the cluster center of 1.5 core radii. A Keplerian orbit has been determined from the periodic variations in the barycentric pulse period of PSR B1516 + 02B. This initial orbital model allows for a TOA model to be constructed from 236 integrations of 23 minutes each, with individual TOA uncertainties of $200 \mu \mathrm{s}$ (Fig. 1). The resulting 7 day binary orbit indicates that the companion star has a mass in excess of $0.11 M_{\odot}$ (assuming a pulsar mass of $1.35 M_{\odot}$ ). Additional constraints on the nature of the companion star may be obtained from the observed orbital precession. If, because of general relativistic effects, the observed rate of $0.015 \mathrm{yr}^{-1}$ corresponds to a total binary mass of $2.48 M_{\odot}$. A nonparametric estimate of the uncertainty in the rate of orbital precession, obtained through a bootstrap calculation (Efron \& Tibshirani 1993), indicates that the central $3 \sigma$ confidence interval covers the symmetric region from 0.0048 to $0.0254 \mathrm{yr}^{-1}$ or, correspondingly, a total binary mass between 0.45 and 5.5 $M_{\odot}$.

This total mass limit leaves open the question of the mass of the companion star. However, continued observations over the next decade should resolve the issue between a low-mass binary pulsar (LMBP) and a high-mass binary pulsar (HMBP). For example, $1 \mathrm{hr}$ observations taken once per month over the next 12 yr would constrain the companion mass to be greater than the $\sim 0.7 M_{\odot}$ turn-off mass of the cluster at the $3 \sigma$ level of confidence, assuming the currently observed precession rate is the true value and that the pulsar mass is $1.35 M_{\odot}$. Or, conversely, $13 \mathrm{yr}$ of observations will constrain the companion mass to be less than $0.7 M_{\odot}$ with the same level of confidence, assuming a true value of $0.35 M_{\odot}$. The large observed eccentricity, $e=0.14$, is consistent with either the LMBP or HMBP scenarios. For an LMBP, perturbations of an initially circular orbit by passing stars in the dense cluster environment may be responsible for the observed eccentricity (Rasio \& Heggie 1995). Alternatively, the system may have acquired eccentricity at the time of formation or more likely in a threebody exchange as for PSR B2127+11C in the cluster M15 (Phinney \& Sigurdsson 1991). In this scenario, PSR $\mathrm{B} 1516+02 \mathrm{~B}$ was an LMBP that exchanged the low-mass companion for a heavier mass field object. The exchange also results in a velocity kick for the entire system. This scenario explains why PSR B1516+02B, like PSR $\mathrm{B} 2127+11 \mathrm{C}$, is further outside the core radius than the respective isolated cluster pulsars.

PSR B1516+02B is observed to have a negative period derivative that is attributed to the pulsar lying behind the cluster and acceleration toward the center of mass of the cluster. The magnitude of the observed $\dot{P} / P=-4.2$ $\times 10^{-19} \mathrm{~s}^{-1}$ is less than the maximum cluster acceleration, $\max \left|a_{l}\right| / c=3 \times 10^{-18} \mathrm{~s}^{-1}$ at PSR B1516+02B's projected radius (Phinney 1993), implying a minimum characteristic age of $6 \times 10^{9} \mathrm{yr}$ and a maximum magnetic field strength of $4 \times 10^{8} \mathrm{G}$.

\section{SUMMARY}

Timing observations of the two previously discovered millisecond pulsars in the globular cluster M5 have been performed over a time span of $5 \mathrm{yr}$. Both the isolated PSR $\mathrm{B} 1516+02 \mathrm{~A}$ and the binary PSR B1516+02B are found near the core radius of the cluster where acceleration in the gravitational field of M5 contaminates the observed period derivatives. In particular, the apparent "spin-up" of PSR $\mathrm{B} 1516+02 \mathrm{~B}$ is consistent with known cluster parameters and the pulsar lying behind the cluster. In addition, the large eccentricity of PSR B1516+02B is consistent with theoretical calculations of the collective perturbations of passing nearby stars on an initially circular orbit (Rasio \& Heggie 1995).

Further observations of M5 and other clusters should continue to lower the detection flux limit for isolated pulsars, placing useful constraints on the underlying population of faint pulsars in globular clusters. In addition, the nature of the companion star to PSR B1516+02B will be addressed by further observations through a more precise determination of the rate of orbital precession and, hence, a tighter constraint on the total mass of the binary system.

We would like to thank the Arecibo telescope operators for many of the observations. S. R. K. has been supported by grants from NSF, NASA, and the Packard Foundation. A. W. and S. B. A. were supported by NSF grant AST 93-17757, and T. A. P. has been supported by NSF grant AST 90-20787. Arecibo Observatory is operated by Cornell University under contract with the NSF. 


\section{REFERENCES}

Anderson, S. B. 1992, Ph.D. thesis, Caltech

Phinney, E. S., \& Kulkarni, S. R. 1994, ARA\&A, 32, 591

Dewey, R. J., Taylor, J. H., Weisberg, J. M., \& Stokes, G. H. 1985, ApJ, 294, L25

Efron, B., \& Tibshirani, R. J. 1993, An Introduction to the Bootstrap (New York: Chapman \& Hall)

Kulkarni, S. R., Anderson, S. B., Prince, T. A., \& Wolszczan, A. 1991, Nature, 349, 47

Lyne, A. G., Manchester, R. N., \& Taylor, J. H. 1985, MNRAS, 213, 613

National Astronomy and Ionosphere Center. 1989, Arecibo Observatory Users's Manual (Ithaca: Cornell)

Nice, D. J., \& Thorsett, S. E. 1992, ApJ, 397, 249

Phinney, E. S. 1993, in ASP Conf. Ser. 50, Structure and Dynamics of Globular Clusters, ed. S. G. Djorgovski \& G. Meylan (San Francisco: ASP), 141

Phinney, E. S., \& Sigurdsson, S. 1991, Nature, 349, 220

Rasio, F. A., \& Heggie, D. C. 1995, ApJ, 445, L133

Rickett, B. J. 1990, ARA\&A, 28, 561

Robinson, C., Lyne, A. G., Manchester, R. N., Bailes, M., D’Amico, N., \& Johnston, S. 1995, MNRAS, 274, 547

Shawl, S. J., \& White, R. E. 1986, AJ, 91, 312

Taylor, J. H., \& Cordes, J. M. 1993, ApJ, 411, 674

Taylor, J. H., \& Weisberg, J. M. 1989, ApJ, 345, 434

Wolszczan, A., Anderson, S., Kulkarni, S., \& Prince, T. 1989a, IAU Circ. 4880

Wolszczan, A., Kulkarni, S. R., Middleditch, J., Backer, D. C., Fruchter, A. S., \& Dewey, R. J. 1989b, Nature, 337, 531 